\title{
Blockchain Technologies in Electric Energy Systems
}

\author{
Nazreen Junaidi \\ School of Electrical Engineering \\ Faculty of Engineering \\ Universiti Teknologi Malaysia \\ UTM Johor Bahru, Johor, Malaysia \\ nazreenjunaidi@graduate.utm.my
}

\author{
Mohamed Shaaban \\ Department of Electrical and \\ Electronic Engineering \\ Faculty of Engineering, Universiti \\ Malaysia Sarawak \\ Kota Samarahan, Sarawak, Malaysia \\ mshaaban@unimas.my
}

\author{
Md Pauzi Abdullah \\ School of Electrical Engineering, \\ Faculty of Engineering \\ Universiti Teknologi Malaysia \\ UTM Johor Bahru, Johor, Malaysia \\ mpauzi@utm.my
}

\begin{abstract}
Demand response (DR) programs development have been currently capturing significant interest by the utilities with the intention to match generation and demand in a more efficient way. The participation of DR programs in electricity markets has increased and has benefitted not only individual consumers but also the market as a whole. In addition, Blockchain and artificial intelligence are emerging technologies that have attracted significant attention from the industries and the academic community. Due to the impact of both technologies on energy systems, applications of blockchain technology in the energy field is explored, in this paper, because of its characteristics and the development needs of the energy Internet realm. An overview of demand response and blockchain technology that covers their types, enabling technologies, worldwide experiences, benefits and challenges are presented. Furthermore, techniques used in blockchain realization are also discussed. Finally, the paper winds up presenting a conceptual framework of blockchained-based proxy demand resources.
\end{abstract}

Keywords - Artificial intelligence, Blockchain technology Demand response, Electric Energy Systems.

\section{INTRODUCTION}

In future energy systems, it is important to increase consumer participation, including demand response (DR) and energy efficiency measures. With the implementation of $\mathrm{DR}$, it is expected to be a cost-effective component to balance grids with large shares of irregular electricity generation [1]. Due to some factors such as increased fuel costs, variability of renewable energy sources, legislative and regulatory pressures and increased customer sensitivity to energy costs, the electric utility industry is considering demand-side options as potential alternative to traditional resource planning.

DR refers to the changes in electricity usage by end-use customers from their normal consumption patterns in

2015 causing power outage affecting the end users to about 225,000 people for many hours [6]. Cyber-attacks create massive losses to intellectual property, business intelligence, the economy, raise security costs, harm to the credibility of an organization, and interrupt work flow. [7]. Furthermore, these attacks could lead to mislead decision making in the control centre causing system disturbances, financial loss or even more serious power outage. Hence, a response to changes in the price of electricity over time [2]. Several forms of DR systems are provided worldwide; some include pre-contracted agreements whereby customers minimize their load in response to signals obtained from the system operator (or aggregator), while others include customers deciding, often on the basis of price signals, whether or not to engage in a demand response opportunity; Some are driven by economic factors, others by reliability concerns. The benefits of DR programs in electricity market context were addressed in the literature [2][3]. DR was classified into four main categories: participant, marketwide, reliability, and market performance benefits [2]. However, the implementation of DR programs are realized by smart meters as well as Advanced Metering Infrastructure (AMI), communication protocols and standards [4]. With the development of advanced metering infrastructure (AMI), communication between system operator and end-use customers enables the demand to be adjusted / curtailed / shifted with respect to the state of the system to ensure reliability. However, as DR is relying heavily on information communication systems and its advanced technologies for their operations, therefore it is potentially more vulnerable to cyberattacks.

Over the past decades, power utilities and grids have become the target of choice of cyberattacks given their vulnerability and the massive economic and social disruption caused by a widespread and lengthy loss of electricity. According to the latest memo on contingency planning from the Foreign Relations Council said that "disabling or otherwise interfering with the power grid in a significant way could seriously harm the United States" [5]. Several cyberattacks have been reported recently against power utilities and grids. The severity of the attacks is also increasing. For example, the world first successful cyberattack on Ukraine's power grid took place in December new disruptive technology paradigm is needed to overcome this problem.

Blockchain technology has been gaining significant momentum recently from a wide range of industries; finance, health care, utilities and the government sector. This is because it offers alluring premise of decentralization, privacy and trust [8]. Furthermore, it provides transparent, user-friendly applications required for energy end users to engage in the energy consumption process. With blockchain 